\title{
Sorting Through the Heterogeneity of Recurrent Melanoma
}

\author{
Lisa K. Jacobs, MD, Julie R. Lange, MD, and Charles M. Balch, MD \\ Departments of Surgery and Oncology, Johns Hopkins Medical Institutions, Baltimore, MD, USA
}

A critical component of managing oncology patients is understanding the natural history of their cancer and identifying those features of the tumor and the patient that predict the metastatic process and survival outcome. The clinical behavior of recurrent or metastatic melanoma is remarkably heterogeneous. In this issue of the Annals of Surgical Oncology, Francken, Thompson and colleagues from the Sydney Melanoma Unit have made yet another valuable contribution to the literature with their assessment of survival outcomes, and independent predictors of survival outcomes in patients with recurrent melanoma. ${ }^{1}$ Most studies of prognostic factors in melanoma patients have examined outcomes from the time of the original diagnosis. ${ }^{2,3}$ This study analyzes prognostic factors predicting metastatic behavior and survival outcome starting with the time of first relapse at different anatomic sites. The ability to predict prognosis from the time of relapse is of critical importance. Better understanding of expected prognosis in patients with recurrent melanoma allows the clinician to make better choices in disease evaluation and treatment, and most importantly for the future of melanoma care, an ability to categorize patients with recurrent disease into groups with more homogeneous prognoses will lead to better design of clinical trials of systemic therapies, hopefully hastening the time when we will have more effective therapies for our patients with advanced disease.

Knowing how to sort through these variables, and reconcile differences in results from the literature, can

Published online March 5, 2008.

Address correspondence and reprint requests to: Lisa K. Jacobs, MD; E-mail: ljacob14@jhmi.edu

Published by Springer Science+Business Media, LLC $\odot 2008$ The Society of Surgical Oncology, Inc. be a difficult, if not daunting, task. Understanding that these clinical and pathological factors are but crude surrogates of more fundamental biological predictors of melanoma behavior and survival outcomes would provide the reader with a more unifying conceptual way of thinking about this subject. Thus, we could partition the factors described in this study by Francken et al. into three components: (1) the biological characteristics of the primary melanoma, (2) the hosts (i.e., patient) systemic responses to contain metastatic growth (such as immunological responses), and (3) the ability of a recurrent or metastatic melanoma to grow in a particular microenvironment.

Using this conceptual framework, one could categorize melanoma patients along a gradation of metastatic potential for primary melanomas based upon whether they are well differentiated and thus would have a low biological capability for completing the metastatic cascade and growing in another microenvironment or are poorly differentiated and thus would have mutational changes that would allow them to grow in a microenvironment for which they were not genetically programmed to grow. There is a large scale of melanoma cell dedifferentiation, each with differing metastatic risk, between these two extremes. Within this conceptual model, it is evident that features of a poorly differentiated primary melanoma - especially melanoma ulceration and high mitotic rate - would be associated with increased metastatic risk.

In the publication by Frankel, the site of first recurrence was a valuable focus of their prognostic factors analysis. ${ }^{1}$ Among 873 patients who relapsed after treatment (between the years 1960 and 2002), the initial site of recurrence was local in $11 \%$, intralymphatic (or in transit) in $10 \%$, regional lymph 
nodes in $34 \%$, and distant in $45 \%$ of patients. By far the most important predictor of survival was location of the recurrence. Following first recurrences, the median survival time was 1.3 years, and varied widely according to the site of first recurrence. Local recurrences had the best prognosis; regional metastases, distant skin, lymph nodes, and lung metastases were intermediate; and all other visceral sites had the worst prognosis. The authors attributed the better prognosis of lung metastases to lead-time bias (due to more aggressive screening) or due to the benefit of metastectomy. An additional possibility is that the metastases that grew in a lung microenvironment might be more indolent in their growth potential compared to metastases that select to grow in other microenvironments, such as liver or brain.

The age of the patient was an independent predictor of survival outcome in this study and indeed in almost all studies of melanoma prognosis. ${ }^{2-4}$ Survival was better for patients $<50$ years of age presenting with locoregional recurrences, but not for those with distant metastases. Increasing age may be a simple but crude surrogate for declining host immunological factors or other host defense mechanism. It is also possible that melanoma in the elderly has different biological characteristics, just as acral lentigo maligna has a different mutational signature compared to solar induced melanomas. Thus, primary melanomas in the elderly tend to have more features of a poorly differentiated melanoma, have a lower rate of nodal metastases, but paradoxically, a higher mortality rate overall.

One of the most interesting findings in this study was that the time period during which a recurrence was diagnosed correlated with survival rates (i.e., comparing the 1960-1970 to the 1990-2002 time periods). This was particularly notable for patients with distant metastasis. Why were survival rates increasing as a function of the decade in which they were diagnosed? Was it better treatment or lead-time bias because of better staging? Equally interesting was the observation that the disease-free interval prior to first relapse was not statistically significant.

For clinical oncologists who are consulting with a melanoma patient after a first relapse, this information is vital to formulate: (1) the stage of disease, (2) the intensity of the metastatic evaluation, (3) the predicted incidence of metastatic disease, (4) a treatment plan that calibrates the combination and se- quence of modalities with the biological aggressiveness of the disease, and (5) predicted survival rates after treatment when counseling the patient during follow-up evaluations. Our criteria for staging melanoma also depend upon knowing the most relevant predictive or prognostic factors of survival outcome. For the clinical investigator, knowing the most independent and dominant prognostic factors is essential in both the design of the clinical trial and interpreting the results. Only with this knowledge can one reliably distinguish between the impact of the treatment under study and the survival outcome based on the natural history of the metastatic process that varies among patients. Otherwise, treatment differences, or lack thereof, may be influenced more by the mix of the patient's prognostic factors than by the treatment effect being studied.

The results from Francken, Thompson, and colleagues once again demonstrate that the disease we refer to as "metastatic melanoma" is actually a very diverse and heterogeneous collection of clinical presentations. Understanding this heterogeneity and taking it into account is important to both the melanoma clinician and the melanoma investigator. In the past, melanoma has been labeled as unpredictable. Actually, the opposite is true as we learn to sort systematically through the multiple characteristics of a very heterogeneous disease. This article from the Sydney Melanoma Unit is a valuable step in that journey to better understand the natural history of metastatic melanoma.

\section{REFERENCES}

1. Francken, AB, Accortt, NA, Shaw, HM, et al. Prognosis and determinants of outcome following locoregional or distant metastases in patients with cutaneous melanoma. Ann Surg Oncol 2008; in press.

2. Balch CM, Soong SJ, Murad TM, et al. A multifactorial analysis of melanoma. III. Prognostic factors in melanoma patients with lymph node metastases (stage II). Ann Surg 1981; 193:377-88.

3. Balch CM, Soong SJ, Murad TM, et al. A multifactorial analysis of melanoma. IV. Prognostic factors in 200 melanoma patients with distant metastases (stage III). J Clin Oncol 1983; 1:126-34.

4. Balch CM, Soong SJ, Gershenwald JE, et al. Prognostic factors analysis of 17,600 melanoma patients: validation of the American Joint Committee on Cancer melanoma staging system. J Clin Oncol 2001; 19:3622-34. 